\title{
A Consistent Approach to Approximate Lie Symmetries of Differential Equations
}

\author{
R. D. Salvo, M. Gorgone and F. Oliveri \\ Department of Mathematical and Computer Sciences, \\ Physical Sciences and Earth Sciences, University of Messina \\ Viale F. Stagno d'Alcontres 31, 98166 Messina, Italy \\ rdisalvo@unime.it; mgorgone@unime.it; foliveri@unime.it \\ Published in Nonlinear Dyn. 91, 371-386 (2018).
}

\begin{abstract}
Lie theory of continuous transformations provides a unified and powerful approach for handling differential equations. Unfortunately, any small perturbation of an equation usually destroys some important symmetries, and this reduces the applicability of Lie group methods to differential equations arising in concrete applications. On the other hand, differential equations containing small terms are commonly and successfully investigated by means of perturbative techniques. Therefore, it is desirable to combine Lie group methods with perturbation analysis, i.e., to establish an approximate symmetry theory. There are two widely used approaches to approximate symmetries: the one proposed in 1988 by Baikov, Gazizov and Ibragimov, and the one introduced in 1989 by Fushchich and Shtelen. Moreover, some variations of the Fushchich-Shtelen method have been proposed with the aim of reducing the length of computations. Here, we propose a new approach that is consistent with perturbation theory and allows to extend all the relevant features of Lie group analysis to an approximate context. Some applications are also presented.
\end{abstract}

Keywords. Differential equations; Approximate Lie symmetries; Perturbation techniques 


\section{Introduction}

The study of Lie continuous transformation groups of differential equations [1, 2, 3, 4, 5, 6, 7, 8, 9, 10] represents a general algorithmic approach to differential equations that, besides its intrinsic theoretical interest (e.g., for classifying differential equations according to the admitted symmetries), plays a fundamental role from a geometrical or analytical viewpoint. In fact, the exploitation of symmetries of differential equations often leads to their simplification.

For ordinary differential equations, the knowledge of their symmetries allows us to algorithmically lower their order or, possibly, reduce them to quadrature. For partial differential equations, the symmetries suggest how to combine the dependent and independent variables in such a way a reduction of their dimension arises, and special (invariant) solutions [3, 4, 11, 12, 13, 14, 15] of initial and boundary value problems can be determined; symmetries play also a role in deriving conserved quantities, or in the algorithmic construction of invertible point transformations linking different differential equations that turn out to be equivalent [9, 16, 17, 18, 19, 20, 21, 22, 23, 24, 25, 26].

Unfortunately, any small perturbation in a differential equation has the - often dramatic - effect of destroying many useful symmetries, and this limits the applicability of Lie group methods to concrete problems where equations involving terms of different order of magnitude may occur. To overcome this inconvenient, some approximate symmetry theories have been proposed in order to deal with differential equations involving small terms, and the notion of approximate invariance has been introduced.

The first, illuminating, paper was by Baikov, Gazizov and Ibragimov [27] (see also [28]), who in 1988 proposed to expand in a perturbation series the Lie generator in order to have an approximate generator. The resulting theory is quite elegant since all the useful properties of exact Lie symmetries can be adapted in the approximate sense: reduction of order of ordinary differential equations, approximately invariant solutions, approximate conservation laws, etc. Since its introduction, this approach has been applied to many physical models [29, 30, 31, 32, 33, 34, 35, 36, 37, 38, 39]. Nevertheless, the expanded generator is not consistent with the principles of perturbation analysis [40] because the dependent variables are not expanded. This implies that in several examples the approximately invariant solutions that are found with this method are not the most general ones. In [41, 42], the approach proposed in [27] has been applied to the investigation of asymptotic waves in dissipative systems by expanding in a perturbation series both the Lie generator and the dependent variables. 
In 1989, Fushchich and Shtelen [43] proposed a different approach. The dependent variables are expanded in a series as done in usual perturbation analysis; terms are then separated at each order of approximation, and a system of equations to be solved in a hierarchy is obtained. This resulting system is assumed to be coupled, and the approximate symmetries of the original equations are defined as the exact symmetries of the equations obtained from separation. This approach has an obvious simple and coherent basis. Per contra, a lot of algebra (especially for higher order perturbations) is needed; moreover, the basic assumption of a fully coupled system is too strong, since the equations at a level are not influenced by those at higher levels. In addition, there is no possibility to work in a hierarchy: for instance, if one computes first order approximate symmetries, and then searches for second order approximate symmetries, all the work must be done from the very beginning. Applications of this method to various equations can be found, for instance, in the papers [35, 44, 45, 46, 47].

In 2004, Pakdemirli et al. [33] compared the two different approaches, and proposed a third method as a variation of Fushchich-Shtelen one by removing the assumption of a fully coupled system. This method applies to differential equations having the form

$$
\mathscr{L}(\mathbf{u})+\varepsilon \mathscr{N}(\mathbf{u})=0,
$$

where $\mathscr{L}(\mathbf{u})$ and $\mathscr{N}(\mathbf{u})$ are a linear and a nonlinear differential operator, respectively. Expanding the dependent variables,

$$
\mathbf{u}=\mathbf{u}_{(0)}+\varepsilon \mathbf{u}_{(1)}+\varepsilon^{2} \mathbf{u}_{(2)}+\ldots
$$

equation (1) provides

$$
\begin{aligned}
& \mathscr{L}\left(\mathbf{u}_{(0)}\right)=0=h_{0}(\mathbf{x}), \\
& \mathscr{L}\left(\mathbf{u}_{(1)}\right)=-\mathscr{N}\left(\mathbf{u}_{(0)}\right)=h_{1}(\mathbf{x}), \\
& \mathscr{L}\left(\mathbf{u}_{(2)}\right)=-\mathscr{N}\left(\mathbf{u}_{(0)}, \mathbf{u}_{(1)}\right)=h_{2}(\mathbf{x}),
\end{aligned}
$$

Since the differential operators in the left-hand sides of all the equations are the same, and the right-hand sides can be considered as arbitrary functions of the independent variables which are determined sequentially starting from the first equation, the approximate symmetries of the nonlinear equation (1) are defined as the exact symmetries of the linear nonhomogeneous equation

$$
\mathscr{L}(\mathbf{u})=h(\mathbf{x}),
$$


with $h(\mathbf{x})$ considered as an arbitrary function. Due to the circumstance that the functions $h_{i}(\mathbf{x})$ in (3) are in fact known functions, substituting these forms into the general expression of the symmetries of equation (4), one obtains the symmetries at each level of approximation. The involved algebra is much less than that required by Fushchich-Shtelen method, and it is possible to work in a hierarchy; nevertheless, the method is not general.

It is well known that searching for Lie symmetries of differential equations requires a lot of long and tedious, though straightforward, calculations. Nevertheless, many symbolic packages do exist doing almost automatically the required work (see [4, 6, 48, 49, 50], and references therein). The computation of approximate Lie symmetries of differential equations imposes additional computational costs. In a recent paper [51], Jefferson and Carminati described the MAPLE package ASP (Automated Symmetry Package), which is an add-on to the MAPLE symmetry package DESOLVII [52], for automating the above three methods of determining approximate symmetries for differential equations.

A further variant of Fushchich-Shtelen method has been proposed in [53] for the analysis of a third order partial differential equation describing one-dimensional wave propagation in nonlinear dissipative media:

$$
w_{t t}-f\left(w_{x}\right) w_{x x}=\varepsilon w_{x x t},
$$

where $f\left(w_{x}\right)$ is an arbitrary function and the subscripts $t$ and $x$ denote partial derivatives. Inserting the expansion of the dependent variable in power series of $\varepsilon$,

$$
w(t, x, \varepsilon)=w_{0}(t, x)+\varepsilon w_{1}(t, x)+O\left(\varepsilon^{2}\right),
$$

into equation (5), and separating terms with different powers in $\varepsilon$, an approximate system is found. Then, the approximate symmetries of equation (5) are defined as the (exact) symmetries of the approximate system through the Lie group generated by

$$
\begin{aligned}
\Xi & =\xi_{0}^{1}\left(t, x, w_{0}\right) \frac{\partial}{\partial t}+\xi_{0}^{2}\left(t, x, w_{0}\right) \frac{\partial}{\partial x}+\eta_{0}\left(t, x, w_{0}\right) \frac{\partial}{\partial w_{0}} \\
& +\left(\eta_{10}\left(t, x, w_{0}\right)+\eta_{11}\left(t, x, w_{0}\right) w_{1}\right) \frac{\partial}{\partial w_{1}} .
\end{aligned}
$$

This approach allows to work in a hierarchy; per contra, there is not a full mix between Lie group methods and perturbation analysis (as in the case of FushchichShtelen method). Applications of this approach can be found in [54, 55].

The aim of this paper is to propose an approximate symmetry theory which is consistent with perturbative analysis and inherits the relevant properties of ex- 
act Lie symmetries of differential equations. More precisely, the dependent variables are expanded in power series of the small parameter as done in classical perturbative analysis; then, instead of considering the approximate symmetries as the exact symmetries of the approximate system (as done in Fushchich-Shtelen method), the consequent expansion of the Lie generator is constructed, and the approximate invariance with respect to the approximate Lie generator is introduced, as in Baikov-Gazizov-Ibragimov method. Of course, the method requires more computations than that required for determining exact Lie symmetries; nevertheless, a general Reduce [56] package (ReLie, [57]), written by one of the authors (F. O.), is able to compute - besides exact, conditional and contact symmetries as well as equivalence transformations of differential equations - approximate Lie symmetries as introduced in this paper.

The paper is organized as follows. Section 2 is devoted to fix the notation, define the approximate one-parameter Lie groups of transformations, and properly state all the relevant properties of classical Lie groups of transformations in the approximate framework. Section 3 deals more specifically with approximate Lie symmetries of differential equations. In Section 4, by means of some examples, the use of approximate Lie symmetries to lower the order of an ordinary differential equation, and determine approximately invariant solutions of partial differential equations is shown. Finally, Section 5 contains our conclusions.

\section{Approximate one-parameter Lie groups}

We begin this Section by introducing the necessary notation in order to define approximate one-parameter Lie groups of transformations.

Let $f(\mathbf{z}, \varepsilon)$ be a $C^{\infty}$ function depending on $\mathbf{z} \equiv\left(z_{1}, \ldots, z_{N}\right) \in D \subseteq \mathbb{R}^{N}$ and the small parameter $\varepsilon \in \mathbb{R}$; in the following, we will consider such kind of functions locally in a neighborhood of $\varepsilon=0$.

Let us expand $\mathbf{z}$ in power series of $\varepsilon$,

$$
\mathbf{z}=\mathbf{z}_{(0)}+\varepsilon \mathbf{z}_{(1)}+\cdots+\varepsilon^{p} \mathbf{z}_{(p)}+O\left(\varepsilon^{p+1}\right),
$$

with $\mathbf{z}_{(k)} \equiv\left(z_{(k) 1}, \ldots, z_{(k) N}\right)$, whereupon

$$
f(\mathbf{z}, \varepsilon)=\left.\sum_{k=0}^{p} \sum_{|\sigma|=k} \frac{\varepsilon^{\sigma_{0}}}{\sigma_{0} !}\left(\prod_{i=1}^{N} \frac{\left(z_{i}-z_{(0) i}\right)^{\sigma_{i}}}{\sigma_{i} !}\right) \frac{\partial^{|\sigma|} f(\mathbf{z}, \varepsilon)}{\partial \varepsilon^{\sigma_{0}} \partial z_{1}^{\sigma_{1}} \cdots \partial z_{N}^{\sigma_{N}}}\right|_{\varepsilon=0}+O\left(\varepsilon^{p+1}\right),
$$


$\sigma$ being the multi-index $\left(\sigma_{0}, \sigma_{1}, \ldots, \sigma_{N}\right)$, and $|\sigma|=\sigma_{0}+\sigma_{1}+\cdots+\sigma_{N}$.

By means of the positions

$$
\begin{aligned}
f_{(0)}\left(\mathbf{z}_{(0)}\right) & =\left.f(\mathbf{z}, \varepsilon)\right|_{\varepsilon=0}, \\
f_{(k)}\left(\mathbf{z}_{(0)}\right) & =\left.\frac{\partial^{k} f(\mathbf{z}, \varepsilon)}{\partial \varepsilon^{k}}\right|_{\varepsilon=0},
\end{aligned}
$$

since it is

$$
\left.\frac{\partial^{|\sigma|} f(\mathbf{z}, \varepsilon)}{\partial \varepsilon^{\sigma_{0}} \partial z_{1}^{\sigma_{1}} \cdots \partial z_{N}^{\sigma_{N}}}\right|_{\varepsilon=0}=\frac{\partial^{|\sigma|-\sigma_{0}} f_{\left(\sigma_{0}\right)}\left(\mathbf{z}_{(0)}\right)}{\partial z_{(0) 1}^{\sigma_{1}} \cdots \partial z_{(0) N}^{\sigma_{N}}}
$$

we have:

$$
f(\mathbf{z}, \varepsilon)=\sum_{k=0}^{p} \sum_{|\sigma|=k} \frac{\varepsilon^{\sigma_{0}}}{\sigma_{0} !}\left(\prod_{i=1}^{N} \frac{\left(z_{i}-z_{(0) i}\right)^{\sigma_{i}}}{\sigma_{i} !}\right) \frac{\partial^{|\sigma|-\sigma_{0}} f_{\left(\sigma_{0}\right)}\left(\mathbf{z}_{(0)}\right)}{\partial z_{(0) 1}^{\sigma_{1}} \cdots \partial z_{(0) N}^{\sigma_{N}}}+O\left(\varepsilon^{p+1}\right) ;
$$

therefore, the expansion in power series of $\varepsilon$ is characterized (up to the order $p$ in $\varepsilon$ ) by $p+1$ functions of $\mathbf{z}_{(0)}$. Such an expansion can be written as

$$
f(\mathbf{z}, \varepsilon)=\sum_{k=0}^{p} \varepsilon^{k} \widetilde{f}_{(k)}\left(\mathbf{z}_{(0)}, \ldots, \mathbf{z}_{(k)}\right)+O\left(\varepsilon^{p+1}\right),
$$

where $\widetilde{f}_{(k)}(k>0)$ are suitable polynomials in $\mathbf{z}_{(1)}, \ldots, \mathbf{z}_{(k)}$ with coefficients given by $f_{(0)}\left(\mathbf{z}_{(0)}\right), \ldots, f_{(k)}\left(\mathbf{z}_{(0)}\right)$ and their derivatives with respect to $\mathbf{z}_{(0)}$. More precisely, the functions $\widetilde{f}_{(k)}$ are defined as follows:

$$
\begin{aligned}
& \widetilde{f}_{(0)}=f_{(0)}, \\
& \widetilde{f}_{(k+1)}=\frac{1}{k+1} \mathscr{R}\left[\widetilde{f}_{(k)}\right],
\end{aligned}
$$

$\mathscr{R}$ being a linear recursion operator satisfying product rule of derivatives and such that

$$
\begin{aligned}
& \mathscr{R}\left[\frac{\partial^{|\tau|} f_{(k)}\left(\mathbf{z}_{(0)}\right)}{\partial z_{(0) 1}^{\tau_{1}} \ldots \partial z_{(0) N}^{\tau_{N}}}\right]=\frac{\partial^{|\tau|} f_{(k+1)}\left(\mathbf{z}_{(0)}\right)}{\partial z_{(0) 1}^{\tau_{1}} \ldots \partial z_{(0) N}^{\tau_{N}}} \\
&+\sum_{i=1}^{N} \frac{\partial}{\partial z_{(0) i}}\left(\frac{\partial^{|\tau|} f_{(k)}\left(\mathbf{z}_{(0)}\right)}{\partial z_{(0) 1}^{\tau_{1}} \ldots \partial z_{(0) N}^{\tau_{N}}}\right) z_{(1) i}, \\
& \mathscr{R}\left[z_{(k) j}\right]=(k+1) z_{(k+1) j},
\end{aligned}
$$

where $k \geq 0, j=1, \ldots, N,|\tau|=\tau_{1}+\cdots+\tau_{N}$. 
Definition 1. Given two smooth functions $f(\mathbf{z}, \varepsilon)$ and $g(\mathbf{z}, \varepsilon)$, and fixing a positive integer $p$, we will write

$$
f(\mathbf{z}, \varepsilon) \stackrel{p}{\approx} g(\mathbf{z}, \varepsilon)
$$

when it is

$$
f(\mathbf{z}, \varepsilon)=g(\mathbf{z}, \varepsilon)+O\left(\varepsilon^{p+1}\right) .
$$

This means that $g(\mathbf{z}, \varepsilon)$ has the same Taylor expansion as $f(\mathbf{z}, \varepsilon)$ up to the order $p$ in $\varepsilon$. In the rest of the paper, we shall use the simplest notation $\approx$ instead of $\underset{\sim}{\approx}$.

Definition 2 (Approximate one-parameter Lie groups). Let $D \subseteq \mathbb{R}^{N}$ be an open domain, $E \subseteq \mathbb{R}$ an interval containing zero, $S \subseteq \mathbb{R}$ another interval (bounded or unbounded) containing zero and such that $\forall a, b \in S, a+b \in S$. The set of transformations

$$
\begin{aligned}
& \mathbf{Z}: D \times E \times S \rightarrow D, \\
& (\mathbf{z}, \varepsilon, a) \mapsto \mathbf{z}^{\star}=\mathbf{Z}(\mathbf{z}, \varepsilon ; a),
\end{aligned}
$$

depending on the parameter $a$, where $\varepsilon \in E$ is small, is a one-parameter (a) Lie group of transformations if

1. $\mathbf{Z}(\mathbf{Z}(\mathbf{z}, \varepsilon ; a), \varepsilon ; b)=\mathbf{Z}(\mathbf{z}, \varepsilon ; a+b)$;

2. $\mathbf{Z}(\mathbf{z}, \varepsilon ; 0)=\mathbf{z}$;

3. for each value of the parameter $a \in S$ and each $\varepsilon \in E$ the transformations are one-to-one onto D;

4. $\mathbf{Z}$ is $C^{\infty}$ with respect to $\mathbf{z} \in D$ and $\varepsilon \in E$, and analytic with respect to the parameter $a$.

Let us fix a positive integer $p$, and expand $\mathbf{z}$ in power series of $\varepsilon$,

$$
\mathbf{z}=\mathbf{z}_{(0)}+\varepsilon \mathbf{z}_{(1)}+\cdots+\varepsilon^{p} \mathbf{z}_{(p)}+O\left(\varepsilon^{p+1}\right),
$$

whereupon, by defining

$$
\begin{aligned}
& \mathbf{Z}_{(0)}\left(\mathbf{z}_{(0)}\right)=\left.\mathbf{Z}(\mathbf{z}, \varepsilon)\right|_{\varepsilon=0}, \\
& \mathbf{Z}_{(k)}\left(\mathbf{z}_{(0)}\right)=\left.\frac{\partial^{k} \mathbf{Z}(\mathbf{z}, \boldsymbol{\varepsilon})}{\partial \varepsilon^{k}}\right|_{\varepsilon=0},
\end{aligned}
$$

we have

$$
\mathbf{Z}(\mathbf{z}, \varepsilon ; a)=\sum_{k=0}^{p} \varepsilon^{k} \widetilde{\mathbf{Z}}_{(k)}\left(\mathbf{z}_{(0)}, \ldots, \mathbf{z}_{(k)} ; a\right)+O\left(\varepsilon^{p+1}\right)
$$


along with

$$
\begin{aligned}
& \widetilde{\mathbf{Z}}_{(0)}\left(\mathbf{z}_{(0)}\right)=\mathbf{Z}_{(0)}\left(\mathbf{z}_{(0)}\right), \\
& \widetilde{\mathbf{Z}}_{(k+1)}=\frac{1}{k+1} \mathscr{R}\left[\widetilde{\mathbf{Z}}_{(k)}\right], \quad k \geq 0,
\end{aligned}
$$

where $\mathscr{R}$ is the recursion operator defined in (13).

The transformation

$$
\mathbf{z}^{\star}=\mathbf{Z}(\mathbf{z}, \varepsilon ; a) \approx \sum_{k=0}^{p} \varepsilon^{k} \widetilde{\mathbf{Z}}_{(k)}\left(\mathbf{z}_{(0)}, \ldots, \mathbf{z}_{(k)} ; a\right)
$$

is an approximate one-parameter Lie group with respect to the parameter a if

$$
\mathbf{Z}(\mathbf{z}, \varepsilon ; 0) \approx \mathbf{z}, \quad \mathbf{Z}(\mathbf{Z}(\mathbf{z}, \varepsilon ; a), \varepsilon ; b) \approx \mathbf{Z}(\mathbf{z}, \varepsilon ; a+b),
$$

and the condition $\mathbf{Z}(\mathbf{z}, \varepsilon ; a) \approx \mathbf{z}$ for all $\mathbf{z}$ implies $a=0$.

Hereafter we are going to show that all the relevant features of classical Lie groups of transformations can be extended in the approximate sense.

Definition 3 (Generator of an approximate Lie group). The generator of the Lie group of transformations

$$
\mathbf{z}^{\star}=\mathbf{Z}(\mathbf{z}, \varepsilon ; a) \approx \sum_{k=0}^{p} \varepsilon^{k} \widetilde{\mathbf{Z}}_{(k)}\left(\mathbf{z}_{(0)}, \ldots, \mathbf{z}_{(k)} ; a\right)
$$

is given by

$$
\Xi=\sum_{i=1}^{N} \zeta_{i}(\mathbf{z}, \varepsilon) \frac{\partial}{\partial z_{i}}
$$

where

$$
\zeta_{i}(\mathbf{z}, \varepsilon)=\left.\frac{\partial Z_{i}(\mathbf{z}, \varepsilon ; a)}{\partial a}\right|_{a=0} .
$$

Inserting the expansion of $\mathbf{z}$, we have

$$
\zeta_{i}(\mathbf{z}, \varepsilon) \approx \sum_{k=0}^{p} \varepsilon^{k} \widetilde{\zeta}_{(k) i}\left(\mathbf{z}_{(0)}, \ldots, \mathbf{z}_{(k)}\right)
$$

where

$$
\widetilde{\zeta}_{(k) i}\left(\mathbf{z}_{(0)}, \ldots, \mathbf{z}_{(k)}\right)=\left.\frac{\partial \widetilde{Z}_{(k) i}\left(\mathbf{z}_{(0)}, \ldots, \mathbf{z}_{(k)} ; a\right)}{\partial a}\right|_{a=0}
$$


Setting

$$
\begin{aligned}
& \zeta_{(0) i}\left(\mathbf{z}_{(0)}\right)=\left.\zeta_{i}(\mathbf{z}, \varepsilon)\right|_{\varepsilon=0}, \\
& \zeta_{(k) i}\left(\mathbf{z}_{(0)}\right)=\left.\frac{\partial^{k} \zeta_{i}(\mathbf{z}, \varepsilon)}{\partial \varepsilon^{k}}\right|_{\varepsilon=0},
\end{aligned}
$$

it is

$$
\begin{aligned}
& \widetilde{\zeta}_{(0) i}\left(\mathbf{z}_{(0)}\right)=\zeta_{(0) i}\left(\mathbf{z}_{(0)}\right), \\
& \widetilde{\zeta}_{(k+1) i}=\frac{1}{k+1} \mathscr{R}\left[\widetilde{\zeta}_{(k) i}\right],
\end{aligned}
$$

where $\mathscr{R}$ is the recursion operator defined in (13). Therefore, we may write the approximate Lie generator as

$$
\Xi \approx \sum_{k=0}^{p} \varepsilon^{k} \widetilde{\Xi}_{(k)}
$$

with obvious meaning of terms.

Example 1. If $p=1$, the generator of the approximate Lie group of transformations reads

$$
\Xi \approx \sum_{i=1}^{N}\left(\zeta_{(0) i}\left(\mathbf{z}_{0}\right)+\varepsilon\left(\zeta_{(1) i}\left(\mathbf{z}_{0}\right)+\sum_{j=1}^{N} \frac{\partial \zeta_{(0) i}\left(\mathbf{z}_{0}\right)}{\partial z_{(0) j}} z_{(1) j}\right)\right) \frac{\partial}{\partial z_{i}} .
$$

Definition 4 (Approximate Lie's equations). Let

$$
\Xi \approx \sum_{k=0}^{p} \varepsilon^{k} \widetilde{\Xi}_{(k)}
$$

be an approximate Lie generator; the corresponding finite transformation

$$
\mathbf{z}^{\star} \approx \sum_{k=0}^{p} \varepsilon^{k} \widetilde{\mathbf{Z}}_{(k)}\left(\mathbf{z}_{(0)}, \ldots, \mathbf{z}_{(k)} ; a\right)
$$

is recovered by solving the approximate Lie's equations

$$
\begin{array}{rlrl}
\frac{d \mathbf{z}_{(0)}^{\star}}{d a} & =\widetilde{\zeta}_{(0)}\left(\mathbf{z}_{(0)}^{\star}\right), & & \mathbf{z}_{(0)}^{\star}(0)=\mathbf{z}_{(0)}, \\
\frac{d \mathbf{z}_{(k)}^{\star}}{d a}=\widetilde{\zeta}_{(k)}\left(\mathbf{z}_{(0)}^{\star}, \ldots, \mathbf{z}_{(k)}^{\star}\right), & \mathbf{z}_{(k)}^{\star}(0)=\mathbf{z}_{(k)}, & k=1, \ldots, p .
\end{array}
$$


Definition 5 (Invariance of a function). A smooth function

$$
F(\mathbf{z}, \varepsilon) \approx \sum_{k=0}^{p} \varepsilon^{k} \widetilde{F}_{(k)}\left(\mathbf{z}_{(0)}, \ldots, \mathbf{z}_{(k)}\right)
$$

is approximately invariant with respect to

$$
\mathbf{z}^{\star} \approx \sum_{k=0}^{p} \varepsilon^{k} \widetilde{\mathbf{Z}}_{(k)}\left(\mathbf{z}_{(0)}, \ldots, \mathbf{z}_{(k)} ; a\right)
$$

if

$$
F\left(\mathbf{z}^{\star}, \varepsilon\right) \approx F(\mathbf{z}, \varepsilon)
$$

By using the same arguments as those in classical Lie theory, the following theorem about the approximate invariance of a function can be stated.

Theorem 1. Let

$$
\Xi \approx \sum_{k=0}^{p} \varepsilon^{k} \widetilde{\Xi}_{(k)}
$$

be an approximate Lie generator of (36). Then, the function

$$
F(\mathbf{z}, \varepsilon) \approx \sum_{k=0}^{p} \varepsilon^{k} \widetilde{F}_{(k)}\left(\mathbf{z}_{(0)}, \ldots, \mathbf{z}_{(k)}\right)
$$

is approximately invariant with respect to (36) if and only if

$$
\Xi(F(\mathbf{z}, \varepsilon)) \approx 0
$$

Some simple algebra to derive the constraints arising from condition (40) is worth of being explicitly given. It is:

$$
\begin{aligned}
& \Xi(F(\mathbf{z}, \varepsilon)) \approx \sum_{j=0}^{p} \varepsilon^{j}\left(\sum_{i=1}^{N} \widetilde{\zeta}_{(j) i}\left(\mathbf{z}_{(0)}, \ldots, \mathbf{z}_{(j)}\right) \frac{\partial F(\mathbf{z}, \varepsilon)}{\partial z_{i}}\right) \approx \\
& \approx \sum_{j, k=0}^{p} \varepsilon^{j+k}\left(\sum_{i=1}^{N} \widetilde{\zeta}_{(j) i}\left(\mathbf{z}_{(0)}, \ldots, \mathbf{z}_{(j)}\right) \frac{\partial \widetilde{F}_{(k)}\left(\mathbf{z}_{(0)}, \ldots, \mathbf{z}_{(k)}\right)}{\partial z_{(0) i}}\right) \approx \\
& \approx \sum_{k=0}^{p} \varepsilon^{k}\left(\sum_{j=0}^{k} \sum_{i=1}^{N} \widetilde{\zeta}_{(j) i}\left(\mathbf{z}_{(0)}, \ldots, \mathbf{z}_{(j)}\right) \frac{\partial \widetilde{F}_{(k-j)}\left(\mathbf{z}_{(0)}, \ldots, \mathbf{z}_{(k-j)}\right)}{\partial z_{(0) i}}\right) .
\end{aligned}
$$


From (40), taking into account (41), and separating the various powers in $\varepsilon$, we easily obtain the conditions

$$
\left.\sum_{j=0}^{k} \Xi_{(j)} F_{(k-j)}\left(\mathbf{z}_{(0)}\right)\right)=\sum_{j=0}^{k} \sum_{i=1}^{N} \zeta_{(j) i}\left(\mathbf{z}_{(0)}\right) \frac{\partial F_{(k-j)}\left(\mathbf{z}_{(0)}\right)}{\partial z_{(0) i}}=0,
$$

for $k=0, \ldots, p$, where we introduced the reduced operators

$$
\Xi_{(j)}=\sum_{i=1}^{N} \zeta_{(j) i}\left(\mathbf{z}_{0}\right) \frac{\partial}{\partial z_{(0) i}}, \quad j=0, \ldots, p .
$$

Therefore, the approximate invariance condition of a function $F(\mathbf{z}, \varepsilon)$ yields the $p+1$ conditions (42) for the $p+1$ functions characterizing the expansion (up to the order $p$ in $\varepsilon$ ) of the function $F$.

Example 2. Let $p=1$, and $\mathbf{z} \equiv\left(z_{1}, z_{2}\right)$; a smooth function

$$
\begin{aligned}
F(\mathbf{z}, \varepsilon) & \approx F_{0}\left(z_{(0) 1}, z_{(0) 2}\right) \\
& +\varepsilon\left(F_{1}\left(z_{(0) 1}, z_{(0) 2}\right)+\frac{\partial F_{0}\left(z_{(0) 1}, z_{(0) 2}\right)}{\partial z_{(0) 1}} z_{(1) 1}+\frac{\partial F_{0}\left(z_{(0) 1}, z_{(0) 2}\right)}{\partial z_{(0) 2}} z_{(1) 2}\right),
\end{aligned}
$$

is approximately invariant with respect to the Lie generator

$$
\Xi \approx\left(z_{(0) 2}+\varepsilon z_{(1) 2}\right) \frac{\partial}{\partial z_{1}}-\left(z_{(0) 1}+\varepsilon z_{(1) 1}\right) \frac{\partial}{\partial z_{2}},
$$

provided that

$$
\begin{aligned}
& z_{(0) 2} \frac{\partial F_{0}\left(z_{(0) 1}, z_{(0) 2}\right)}{\partial z_{(0) 1}}-z_{(0) 1} \frac{\partial F_{0}\left(z_{(0) 1}, z_{(0) 2}\right)}{\partial z_{(0) 2}}=0, \\
& z_{(0) 2} \frac{\partial F_{1}\left(z_{(0) 1}, z_{(0) 2}\right)}{\partial z_{(0) 1}}-z_{(0) 1} \frac{\partial F_{1}\left(z_{(0) 1}, z_{(0) 2}\right)}{\partial z_{(0) 2}}=0,
\end{aligned}
$$

whereupon it is

$$
F\left(z_{1}, z_{2}\right) \approx F_{0}\left(r_{0}^{2}\right)+\varepsilon\left(F_{1}\left(r_{0}^{2}\right)+2\left(z_{(0) 1} z_{(1) 1}+z_{(0) 2} z_{(1) 2}\right) F_{0}^{\prime}\left(r_{0}^{2}\right)\right),
$$

$F_{0}$ and $F_{1}$ being arbitrary functions of $r_{0}^{2}=z_{(0) 1}^{2}+z_{(0) 2}^{2}$, and the prime' denoting the differentiation with respect to the argument. 
Definition 6 (Invariance of an equation). The equation

$$
F(\mathbf{z}, \varepsilon) \approx \sum_{k=0}^{p} \varepsilon^{k} \widetilde{F}_{(k)}\left(\mathbf{z}_{(0)}, \ldots, \mathbf{z}_{(k)}\right)=0
$$

is approximately invariant with respect to (36) if

$$
F\left(\mathbf{z}^{\star}, \varepsilon\right) \approx 0 \quad \text { when } F(\mathbf{z}, \varepsilon) \approx 0 .
$$

Theorem 2. Let

$$
\Xi \approx \sum_{k=0}^{p} \varepsilon^{k} \widetilde{\Xi}_{(k)}
$$

be an approximate Lie generator of (36).

Then, the equation

$$
F(\mathbf{z}, \varepsilon) \approx \sum_{k=0}^{p} \varepsilon^{k} \widetilde{F}_{(k)}\left(\mathbf{z}_{(0)}, \ldots, \mathbf{z}_{(k)}\right)=0
$$

is approximately invariant with respect to (36) if and only if

$$
\Xi(F(\mathbf{z}, \varepsilon)) \approx 0 \quad \text { when } F(\mathbf{z}, \varepsilon) \approx 0,
$$

i.e.,

$$
\Xi(F(\mathbf{z}, \varepsilon)) \approx \lambda(\mathbf{z}, \varepsilon) F(\mathbf{z}, \varepsilon),
$$

$\lambda(\mathbf{z}, \varepsilon)$ being a multiplier.

Definition 7 (Approximate canonical variables). Let

$$
\Xi \approx \sum_{k=0}^{p} \varepsilon^{k} \widetilde{\Xi}_{(k)}
$$

be an approximate Lie generator of (36).

Then,

$$
\mathbf{w}(\mathbf{z}, \varepsilon) \approx \sum_{k=0}^{p} \varepsilon^{k} \widetilde{\mathbf{w}}_{(k)}\left(\mathbf{z}_{(0)}, \ldots, \mathbf{z}_{(k)}\right)
$$

gives a set of approximate canonical variables if

$$
\begin{aligned}
& \Xi\left(w_{i}\right) \approx 0, \quad i=1, \ldots, N-1, \\
& \Xi\left(w_{N}\right) \approx 1 .
\end{aligned}
$$


In terms of the approximate canonical variables, the approximate Lie generator writes as

$$
\Xi \approx \frac{\partial}{\partial w_{N}}
$$

and the approximate Lie group of transformations corresponds to the translation of the variable $w_{N}$ only.

Example 3. Let $\mathbf{z} \equiv\left(z_{1}, z_{2}\right) \in \mathbb{R}^{2}, p=1$ and

$$
\Xi \approx\left(z_{(0) 1}^{2}+\varepsilon\left(2 z_{(0) 1} z_{(1) 1}\right)\right) \frac{\partial}{\partial z_{1}}+\left(z_{(0) 1} z_{(0) 2}+\varepsilon\left(z_{(0) 1} z_{(1) 2}+z_{(0) 2} z_{(1) 1}\right)\right) \frac{\partial}{\partial z_{2}} .
$$

The first order approximate canonical variables

$$
\mathbf{w}(\mathbf{z}, \boldsymbol{\varepsilon}) \approx \mathbf{w}_{(0)}\left(\mathbf{z}_{(0)}\right)+\varepsilon\left(\mathbf{w}_{(1)}\left(\mathbf{z}_{(0)}\right)+\sum_{i=1}^{2} \frac{\partial \mathbf{w}_{(0)}\left(\mathbf{z}_{(0)}\right)}{\partial z_{(0) i}} z_{(1) i}\right)
$$

are determined by solving the following system of partial differential equations:

$$
\begin{aligned}
z_{(0) 1}^{2} \frac{\partial \widetilde{w}_{(0) 1}}{\partial z_{(0) 1}}+z_{(0) 1} z_{(0) 2} \frac{\partial \widetilde{w}_{(0) 1}}{\partial z_{(0) 2}} & =0, & z_{(0) 1}^{2} \frac{\partial \widetilde{w}_{(0) 2}}{\partial z_{(0) 1}}+z_{(0) 1} z_{(0) 2} \frac{\partial \widetilde{w}_{(0) 2}}{\partial z_{(0) 2}} & =1, \\
z_{(0) 1}^{2} \frac{\partial \widetilde{w}_{(1) 1}}{\partial z_{(0) 1}}+z_{(0) 1} z_{(0) 2} \frac{\partial \widetilde{w}_{(1) 1}}{\partial z_{(0) 2}} & =0, & z_{(0) 1}^{2} \frac{\partial \widetilde{w}_{(1) 2}}{\partial z_{(0) 1}}+z_{(0) 1} z_{(0) 2} \frac{\partial \widetilde{w}_{(1) 2}}{\partial z_{(0) 2}} & =0
\end{aligned}
$$

whereupon we have

$$
\begin{aligned}
& w_{1} \approx \frac{z_{(0) 2}}{z_{(0) 1}}+\varepsilon\left(\frac{z_{(0) 2}}{z_{(0) 1}}-\frac{z_{(0) 2} z_{(1) 1}}{z_{(0) 1}^{2}}+\frac{z_{(1) 2}}{z_{(0) 1}}\right), \\
& w_{2} \approx-\frac{1}{z_{(0) 1}}+\varepsilon\left(\frac{z_{(0) 2}}{z_{(0) 1}}+\frac{z_{(1) 1}}{z_{(0) 1}^{2}}\right),
\end{aligned}
$$

and the Lie generator reduces to

$$
\Xi \approx \frac{\partial}{\partial w_{2}}
$$

Definition 8 (Approximate Lie bracket). Let

$$
\begin{array}{ll}
\Xi_{1} \approx \sum_{k=0}^{p} \varepsilon^{k} \widetilde{\Xi}_{1(k)}, & \Xi_{2} \approx \sum_{k=0}^{p} \varepsilon^{k} \widetilde{\Xi}_{2(k)}, \\
\widetilde{\Xi}_{1(k)}=\sum_{j=1}^{N} \widetilde{\zeta}_{1(k) j} \frac{\partial}{\partial z_{j}}, & \widetilde{\Xi}_{2(k)}=\sum_{j=1}^{N} \widetilde{\zeta}_{2(k) j} \frac{\partial}{\partial z_{j}}
\end{array}
$$


be two approximate Lie generators. Their approximate Lie bracket is defined as follows:

$$
\begin{aligned}
& {\left[\Xi_{1}, \Xi_{2}\right] \approx \sum_{k=0}^{p} \varepsilon^{k}\left(\sum_{i=0}^{k}\left[\widetilde{\Xi}_{1(i)}, \widetilde{\Xi}_{2(k-i)}\right]\right) \approx} \\
& \approx \sum_{k=0}^{p} \varepsilon^{k}\left(\sum_{i=0}^{k}\left(\widetilde{\Xi}_{1(i)} \widetilde{\Xi}_{2(k-i)}-\widetilde{\Xi}_{2(k-i)} \widetilde{\Xi}_{1(i)}\right)\right) \approx \\
& \approx \sum_{k=0}^{p} \varepsilon^{k}\left(\sum_{i=0}^{k}\left(\sum_{m=1}^{N}\left(\sum_{j=1}^{N}\left(\widetilde{\zeta}_{1(i) j} \frac{\partial \widetilde{\zeta}_{2(k-i) m}}{\partial z_{(0) j}}-\widetilde{\zeta}_{2(k-i) j} \frac{\partial \widetilde{\zeta}_{1(i) m}}{\partial z_{(0) j}}\right) \frac{\partial}{\partial z_{m}}\right)\right)\right) .
\end{aligned}
$$

The definition of approximate Lie bracket allows us to introduce a structure of approximate Lie algebra, and the following two theorems can be easily proved.

Theorem 3. The set of approximate Lie generators leaving a function approximately invariant has the structure of an approximate Lie algebra.

Theorem 4. The set of approximate Lie generators leaving an equation approximately invariant has the structure of an approximate Lie algebra.

\section{Approximate symmetries of differential equations}

Here we use the results of previous Section in order to define and compute the approximate Lie symmetries of differential equations involving small terms. Within this framework, we distinguish the independent variables $\mathbf{x}$ from the dependent ones $\mathbf{u}$, and limit ourselves to expand only the dependent variables in powers of $\varepsilon$.

Let

$$
\Delta\left(\mathbf{x}, \mathbf{u}, \mathbf{u}^{(r)} ; \varepsilon\right)=0
$$

be a differential equation of order $r$, where $\mathbf{u}^{(r)}$ denotes the set of all derivatives of the dependent variables $\mathbf{u} \in U \subseteq \mathbb{R}^{m}$ with respect to the independent variables $\mathbf{x} \in X \subseteq \mathbb{R}^{n}$ up to the order $r$, involving a small parameter $\varepsilon$.

If one looks for classical Lie point symmetries, in general it is not guaranteed that the infinitesimal generators depend on the parameter $\varepsilon$. Nevertheless, the occurrence of terms involving $\varepsilon$ has dramatic effects since one loses some symmetries admitted by the unperturbed equation

$$
\Delta\left(\mathbf{x}, \mathbf{u}, \mathbf{u}^{(r)} ; 0\right)=0,
$$

as the following examples clearly show. 
Example 4. The second order ordinary differential equation

$$
\frac{d^{2} u}{d x^{2}}+u=0
$$

admits an eight-dimensional Lie algebra of point symmetries spanned by the vector fields:

$$
\begin{array}{llrl}
\Xi_{1} & =\frac{\partial}{\partial x}, & \Xi_{2} & =u \frac{\partial}{\partial u}, \\
\Xi_{3} & =\sin (x) \frac{\partial}{\partial u}, & \Xi_{4} & =\cos (x) \frac{\partial}{\partial u}, \\
\Xi_{5} & =\sin (x) \cos (x) \frac{\partial}{\partial x}-\sin ^{2}(x) u \frac{\partial}{\partial u}, & \Xi_{6} & =\cos (2 x) \frac{\partial}{\partial x}-\sin (2 x) \frac{\partial}{\partial u}, \\
\Xi_{7} & =\cos (x) u \frac{\partial}{\partial x}-\sin (x) u^{2} \frac{\partial}{\partial u}, & \Xi_{8} & =\sin (x) u \frac{\partial}{\partial x}+\cos (x) u \frac{\partial}{\partial u} .
\end{array}
$$

On the contrary, the equation

$$
\frac{d^{2} u}{d x^{2}}+u+\varepsilon u^{3}=0
$$

admits only the exact symmetry generated by $\Xi_{1}$.

Example 5. The Korteweg-deVries equation

$$
\frac{\partial u}{\partial t}+u \frac{\partial u}{\partial x}+\frac{\partial^{3} u}{\partial x^{3}}=0
$$

admits a four-dimensional Lie algebra of exact point symmetries spanned by the vector fields:

$$
\Xi_{1}=\frac{\partial}{\partial t}, \quad \Xi_{2}=\frac{\partial}{\partial x}, \quad \Xi_{3}=t \frac{\partial}{\partial x}+\frac{\partial}{\partial u}, \quad \Xi_{4}=3 t \frac{\partial}{\partial t}+x \frac{\partial}{\partial x}-2 u \frac{\partial}{\partial u} .
$$

On the contrary, by considering the Korteweg-deVries-Burgers equation

$$
\frac{\partial u}{\partial t}+u \frac{\partial u}{\partial x}+\frac{\partial^{3} u}{\partial x^{3}}-\varepsilon \frac{\partial^{2} u}{\partial x^{2}}=0
$$

we lose the scaling group and have only three symmetries:

$$
\Xi_{1}=\frac{\partial}{\partial t}, \quad \Xi_{2}=\frac{\partial}{\partial x}, \quad \Xi_{3}=t \frac{\partial}{\partial x}+\frac{\partial}{\partial u} .
$$


Example 6. The $2 \times 2$ first order quasilinear system

$$
\begin{aligned}
& \frac{\partial u_{1}}{\partial t}+a_{11}\left(u_{1}, u_{2}\right) \frac{\partial u_{1}}{\partial x}+a_{12}\left(u_{1}, u_{2}\right) \frac{\partial u_{2}}{\partial x}=\varepsilon b_{1}\left(u_{1}, u_{2}\right), \\
& \frac{\partial u_{2}}{\partial t}+a_{21}\left(u_{1}, u_{2}\right) \frac{\partial u_{1}}{\partial x}+a_{22}\left(u_{1}, u_{2}\right) \frac{\partial u_{2}}{\partial x}=\varepsilon b_{2}\left(u_{1}, u_{2}\right),
\end{aligned}
$$

where $a_{i j}$ and $b_{i}(i, j=1,2)$ are arbitrary functions of the indicated arguments, does not admit an infinite-dimensional Lie algebra of point symmetries unless it is $\varepsilon=0$. Moreover, also the invariance with respect to a homogeneous scaling of the independent variables, i.e., with respect to the Lie generator

$$
t \frac{\partial}{\partial t}+x \frac{\partial}{\partial x}
$$

is lost when $\varepsilon \neq 0$.

In perturbation theory [40] a differential equation involving small terms is often studied by looking for solutions in the form

$$
\mathbf{u}(\mathbf{x}, \varepsilon)=\sum_{k=0}^{p} \varepsilon^{k} \mathbf{u}_{(k)}(\mathbf{x})+O\left(\varepsilon^{p+1}\right),
$$

whereupon the differential equation writes as

$$
\Delta \approx \sum_{k=0}^{p} \varepsilon^{k} \widetilde{\Delta}_{(k)}\left(\mathbf{x}, \mathbf{u}_{(0)}, \mathbf{u}_{(0)}^{(r)}, \ldots, \mathbf{u}_{(k)}, \mathbf{u}_{(k)}^{(r)}\right)=0 .
$$

Now, let us consider a Lie generator

$$
\Xi=\sum_{i=1}^{n} \xi_{i}(\mathbf{x}, \mathbf{u} ; \varepsilon) \frac{\partial}{\partial x_{i}}+\sum_{\alpha=1}^{m} \eta_{\alpha}(\mathbf{x}, \mathbf{u} ; \varepsilon) \frac{\partial}{\partial u_{\alpha}}
$$

where we assume that the infinitesimals depend on the small parameter $\varepsilon$.

According to the results of previous Section, by using the expansion (70) of the dependent variables only, we have the following expressions for the infinitesimals:

$$
\xi_{i} \approx \sum_{k=0}^{p} \varepsilon^{k} \widetilde{\xi}_{(k) i}, \quad \eta_{\alpha} \approx \sum_{k=0}^{p} \varepsilon^{k} \widetilde{\eta}_{(k) \alpha}
$$


with

$$
\begin{array}{ll}
\widetilde{\xi}_{(0) i}=\xi_{(0) i}=\left.\xi_{i}(\mathbf{x}, \mathbf{u}, \varepsilon)\right|_{\varepsilon=0}, & \widetilde{\eta}_{(0) \alpha}=\eta_{(0) \alpha}=\left.\eta_{\alpha}(\mathbf{x}, \mathbf{u}, \varepsilon)\right|_{\varepsilon=0,}, \\
\widetilde{\xi}_{(k+1) i}=\frac{1}{k+1} \mathscr{R}\left[\widetilde{\xi}_{(k) i}\right], & \widetilde{\eta}_{(k+1) \alpha}=\frac{1}{k+1} \mathscr{R}\left[\widetilde{\eta}_{(k) \alpha}\right],
\end{array}
$$

where, since only the dependent variables are expanded, the recursion operator $\mathscr{R}$ becomes:

$$
\begin{aligned}
& \mathscr{R}\left[\frac{\partial^{|\tau|} f_{(k)}\left(\mathbf{x}, \mathbf{u}_{(0)}\right)}{\partial u_{(0) 1}^{\tau_{1}} \ldots \partial u_{(0) m}^{\tau_{m}}}\right]=\frac{\partial^{|\tau|} f_{(k+1)}\left(\mathbf{x}, \mathbf{u}_{(0)}\right)}{\partial u_{(0) 1}^{\tau_{1}} \ldots \partial u_{(0) m}^{\tau_{m}}} \\
&+\sum_{i=1}^{m} \frac{\partial}{\partial u_{(0) i}}\left(\frac{\partial^{|\tau|} f_{(k)}\left(\mathbf{x}, \mathbf{u}_{(0)}\right)}{\partial u_{(0) 1}^{\tau_{1}} \ldots \partial u_{(0) m}^{\tau_{m}}}\right) u_{(1) i}, \\
& \mathscr{R}\left[u_{(k) j}\right]=(k+1) u_{(k+1) j},
\end{aligned}
$$

for $k \geq 0, j=1, \ldots, m,|\tau|=\tau_{1}+\cdots+\tau_{m}$. Thence, we have an approximate Lie generator

$$
\Xi \approx \sum_{k=0}^{p} \varepsilon^{k} \widetilde{\Xi}_{(k)}
$$

where

$$
\widetilde{\Xi}_{(k)}=\sum_{i=1}^{n} \widetilde{\xi}_{(k) i}\left(\mathbf{x}, \mathbf{u}_{(0)}, \ldots, \mathbf{u}_{(k)}\right) \frac{\partial}{\partial x_{i}}+\sum_{\alpha=1}^{m} \widetilde{\eta}_{(k) \alpha}\left(\mathbf{x}, \mathbf{u}_{(0)}, \ldots, \mathbf{u}_{(k)}\right) \frac{\partial}{\partial u_{\alpha}} .
$$

Since we have to deal with differential equations, we need to prolong the Lie generator to account for the transformation of derivatives. This is done as in classical Lie group analysis of differential equations, i.e., the derivatives are transformed in such a way the contact conditions are preserved. Therefore, we have the prolongations

$$
\begin{aligned}
& \Xi^{(0)}=\Xi, \\
& \Xi^{(r)}=\Xi^{(r-1)}+\sum_{\alpha=1}^{m} \sum_{i_{1}=1}^{n} \ldots \sum_{i_{r}=1}^{n} \eta_{\alpha, i_{1} \ldots i_{r}} \frac{\partial}{\partial \frac{\partial^{r} u_{\alpha}}{\partial x_{i_{1}} \ldots \partial x_{i_{r}}}}, \quad r>0,
\end{aligned}
$$

where

$$
\eta_{\alpha, i_{1} \ldots i_{r}}=\frac{D \eta_{\alpha, i_{1} \ldots i_{r-1}}}{D x_{i_{r}}}-\sum_{k=1}^{n} \frac{D \xi_{k}}{D x_{i_{r}}} \frac{\partial^{r} u_{\alpha}}{\partial x_{i_{1}} \ldots \partial x_{i_{r-1}} \partial x_{k}}
$$


along with the Lie derivative defined as

$$
\frac{D}{D x_{i}}=\frac{\partial}{\partial x_{i}}+\sum_{\alpha=1}^{m}\left(\frac{\partial u_{\alpha}}{\partial x_{i}} \frac{\partial}{\partial u_{\alpha}}+\sum_{j=1}^{n} \frac{\partial^{2} u_{\alpha}}{\partial x_{i} \partial x_{j}} \frac{\partial}{\partial\left(\partial u_{\alpha} / \partial x_{j}\right)}+\cdots\right) .
$$

Of course, in the expression of prolongations, we need to take into account the expansions of $\xi_{i}, \eta_{\alpha}$ and $u_{\alpha}$, and drop the $O\left(\varepsilon^{p+1}\right)$ terms.

Example 7. Let $p=1$, and consider the approximate Lie generator

$$
\begin{aligned}
\Xi & \approx \sum_{i=1}^{n}\left(\xi_{(0) i}+\varepsilon\left(\xi_{(1) i}+\sum_{\beta=1}^{m} \frac{\partial \xi_{(0) i}}{\partial u_{(0) \beta}} u_{(1) \beta}\right)\right) \frac{\partial}{\partial x_{i}} \\
& +\sum_{\alpha=1}^{m}\left(\eta_{(0) \alpha}+\varepsilon\left(\eta_{(1) \alpha}+\sum_{\beta=1}^{m} \frac{\partial \eta_{(0) \alpha}}{\partial u_{(0) \beta}} u_{(1) \beta}\right)\right) \frac{\partial}{\partial u_{\alpha}}
\end{aligned}
$$

where $\xi_{(0) i}, \xi_{(1) i}, \eta_{(0) \alpha}$ and $\eta_{(1) \alpha}$ depend on $\left(\mathbf{x}, \mathbf{u}_{(0)}\right)$. The first order prolongation is

$$
\Xi^{(1)} \approx \Xi+\sum_{\alpha=1}^{m} \sum_{i=1}^{n} \eta_{\alpha, i} \frac{\partial}{\partial \frac{\partial u_{\alpha}}{\partial x_{i}}}
$$

where

$$
\begin{aligned}
\eta_{\alpha, i} & =\frac{D}{D x_{i}}\left(\eta_{(0) \alpha}+\varepsilon\left(\eta_{(1) \alpha}+\sum_{\beta=1}^{m} \frac{\partial \eta_{(0) \alpha}}{\partial u_{(0) \beta}} u_{(1) \beta}\right)\right) \\
& -\sum_{j=1}^{n} \frac{D}{D x_{i}}\left(\xi_{(0) j}+\varepsilon\left(\xi_{(1) j}+\sum_{\beta=1}^{m} \frac{\partial \xi_{(0) j}}{\partial u_{(0) \beta}} u_{(1) \beta}\right)\right)\left(\frac{\partial u_{(0) \alpha}}{\partial x_{j}}+\varepsilon \frac{\partial u_{(1) \alpha}}{\partial x_{j}}\right)
\end{aligned}
$$

with the Lie derivative now defined as

$$
\frac{D}{D x_{i}}=\frac{\partial}{\partial x_{i}}+\sum_{k=0}^{p} \sum_{\alpha=1}^{m}\left(\frac{\partial u_{(k) \alpha}}{\partial x_{i}} \frac{\partial}{\partial u_{(k) \alpha}}+\sum_{j=1}^{n} \frac{\partial^{2} u_{(k) \alpha}}{\partial x_{i} \partial x_{j}} \frac{\partial}{\partial\left(\partial u_{(k) \alpha} / \partial x_{j}\right)}+\cdots\right)
$$

Things go similarly for higher order prolongations.

The approximate (at the order $p$ ) invariance condition of a differential equation reads

$$
\left.\Xi^{(r)} \Delta\right|_{\Delta \approx 0} \approx 0
$$


In the resulting condition we have to insert the expansion of $\mathbf{u}$ in order to obtain the determining equations at the various orders in $\varepsilon$.

The Lie generator $\widetilde{\Xi}_{(0)}$ is always a symmetry of the unperturbed equations $(\varepsilon=0)$; the correction terms $\sum_{k=1}^{p} \varepsilon^{k} \widetilde{\Xi}_{(k)}$ give the deformation of the symmetry due to the terms involving $\varepsilon$.

Remark 1. Not all the symmetries of the unperturbed equations are admitted as the zeroth terms of the approximate symmetries; the symmetries of the unperturbed equations that are the zeroth terms of the approximate symmetries are called stable symmetries [27].

Remark 2. If $\Xi$ is the generator of an approximate Lie point symmetry of a differential equation, $\varepsilon \Xi$ is a generator of an approximate Lie point symmetry too, but the converse is not true in general.

By the same arguments as in classical Lie theory of differential equations, it remains proved the following result.

Theorem 5. The approximate Lie point symmetries of a differential equation are the elements of an approximate Lie algebra.

Let us show some simple examples of first order approximate Lie symmetries admitted by differential equations.

Example 8. The second order ordinary differential equation

$$
\frac{d^{2} u}{d x^{2}}+u+\varepsilon u^{3}=0
$$

admits a nine-dimensional approximate Lie algebra of first order approximate point symmetries spanned by the vector fields:

$$
\begin{array}{ll}
\Xi_{1}=\frac{\partial}{\partial x}, & \Xi_{2}=\varepsilon u_{0} \frac{\partial}{\partial u}, \\
\Xi_{3}=\varepsilon \sin (x) \frac{\partial}{\partial u}, & \Xi_{4}=\varepsilon \cos (x) \frac{\partial}{\partial u}, \\
\Xi_{5}=\varepsilon\left(\cos (2 x) \frac{\partial}{\partial x}-\sin (2 x) u_{0} \frac{\partial}{\partial u}\right), & \Xi_{6}=\varepsilon\left(\sin (2 x) \frac{\partial}{\partial x}+\cos (2 x) u_{0} \frac{\partial}{\partial u}\right), \\
\Xi_{7}=\varepsilon\left(u_{0} \sin (x) \frac{\partial}{\partial x}+u_{0}^{2} \cos (x) \frac{\partial}{\partial u}\right), & \Xi_{8}=\varepsilon\left(u_{0} \cos (x) \frac{\partial}{\partial x}-u_{0}^{2} \sin (x) \frac{\partial}{\partial u}\right), \\
\Xi_{9}=\varepsilon \frac{\partial}{\partial x} . &
\end{array}
$$


Example 9 (KdVB equations). Consider the Korteweg-deVries equation perturbed with the addition of a small dissipative term,

$$
\frac{\partial u}{\partial t}+u \frac{\partial u}{\partial x}+\frac{\partial^{3} u}{\partial x^{3}}-\varepsilon \frac{\partial^{2} u}{\partial x^{2}}=0
$$

The first order approximate symmetries are spanned by the following vector fields:

$$
\begin{aligned}
& \Xi_{1}=\frac{\partial}{\partial t}, \quad \Xi_{2}=\frac{\partial}{\partial x}, \quad \Xi_{3}=t \frac{\partial}{\partial x}+\frac{\partial}{\partial u}, \\
& \Xi_{4}=\varepsilon \frac{\partial}{\partial t}, \quad \Xi_{5}=\varepsilon \frac{\partial}{\partial x}, \quad \Xi_{6}=\varepsilon\left(t \frac{\partial}{\partial x}+\frac{\partial}{\partial u}\right), \\
& \Xi_{7}=\varepsilon\left(3 t \frac{\partial}{\partial t}+x \frac{\partial}{\partial x}-2 u_{0} \frac{\partial}{\partial u}\right) .
\end{aligned}
$$

Analogously, the Burgers equation perturbed with the addition of a small dispersive term,

$$
\frac{\partial u}{\partial t}+u \frac{\partial u}{\partial x}-\frac{\partial^{2} u}{\partial x^{2}}+\varepsilon \frac{\partial^{3} u}{\partial x^{3}}=0
$$

admits the first order approximate symmetries spanned by the following vector fields:

$$
\begin{aligned}
& \Xi_{1}=\frac{\partial}{\partial t}, \quad \Xi_{2}=\frac{\partial}{\partial x}, \quad \Xi_{3}=t \frac{\partial}{\partial x}+\frac{\partial}{\partial u}, \\
& \Xi_{4}=\varepsilon \frac{\partial}{\partial t}, \quad \Xi_{5}=\varepsilon \frac{\partial}{\partial x}, \quad \Xi_{6}=\varepsilon\left(t \frac{\partial}{\partial x}+\frac{\partial}{\partial u}\right), \\
& \Xi_{7}=\varepsilon\left(2 t \frac{\partial}{\partial t}+x \frac{\partial}{\partial x}-u_{0} \frac{\partial}{\partial u}\right), \quad \Xi_{8}=\varepsilon\left(t^{2} \frac{\partial}{\partial t}+t x \frac{\partial}{\partial x}+\left(x-t u_{0}\right) \frac{\partial}{\partial u}\right) .
\end{aligned}
$$

\section{Applications}

The approximate Lie symmetries can be used to lower the order of ordinary differential equations, as well as to compute approximately invariant solutions of partial differential equations. For simplicity, in the following examples we take $p=1$, i.e., we consider first order approximate symmetries. For higher values of $p$ what is only needed is a larger amount of computations. Moreover, since hereafter only scalar differential equations are considered, in order to simplify the notation, the expansion for the unknown $u$ is written as $u=u_{0}+\varepsilon u_{1}+O\left(\varepsilon^{2}\right)$. 


\subsection{Order lowering of an ordinary differential equation}

Here we are going to show how a solvable approximate Lie algebra of symmetries can be used to lower the order of an ordinary differential equation.

Example 10 (Perturbed Blasius equation). Let us consider the equation

$$
\frac{d^{3} u}{d x^{3}}+\frac{1}{2} u \frac{d^{2} u}{d x^{2}}+\varepsilon u \frac{d u}{d x}=0
$$

that for $\varepsilon=0$ is the well known Blasius equation. For $\varepsilon \neq 0$, equation (91) admits only one exact Lie point symmetry, say

$$
\Xi=\frac{\partial}{\partial x}
$$

On the contrary, looking for first order approximate symmetries, equation (91) admits a four-dimensional approximate Lie algebra spanned by the vector fields:

$$
\begin{aligned}
& \Xi_{1}=\frac{\partial}{\partial x}, \quad \Xi_{2}=\varepsilon \frac{\partial}{\partial x}, \quad \Xi_{3}=\varepsilon\left(x \frac{\partial}{\partial x}-u_{0} \frac{\partial}{\partial u}\right), \\
& \Xi_{4}=x \frac{\partial}{\partial x}-u_{0} \frac{\partial}{\partial u}+\varepsilon\left(\frac{x^{2}}{3} \frac{\partial}{\partial x}+\left(8-\frac{2}{3} u_{0} x-u_{1}\right) \frac{\partial}{\partial u}\right) .
\end{aligned}
$$

Let us consider the approximate operators $\widetilde{\Xi}_{1}=\Xi_{1}+\frac{2}{3} \Xi_{3}$ and $\widetilde{\Xi}_{2}=\Xi_{4}$; it is $\left[\widetilde{\Xi}_{1}, \widetilde{\Xi}_{2}\right] \approx \widetilde{\Xi}_{1}$, that means that $\widetilde{\Xi}_{1}$ and $\widetilde{\Xi}_{2}$ span an approximate (at first order) solvable two-dimensional Lie subalgebra. Let us introduce the canonical variables for the operator $\widetilde{\Xi}_{1}$, say

$$
\widetilde{\Xi}_{1}(v)=0, \quad \widetilde{\Xi}_{1}(t)=1,
$$

whereupon

$$
v=u_{0}+\varepsilon\left(\frac{2}{3} u_{0} x+u_{1}\right), \quad t=x-\varepsilon \frac{x^{2}}{3},
$$

and equation (91) becomes

$$
\frac{d^{3} v}{d t^{3}}+\frac{1}{2} v \frac{d^{2} v}{d t^{2}}-4 \varepsilon \frac{d^{2} v}{d t^{2}}=0
$$

The standard substitution $\frac{d v}{d t}=w(v)$ yields, if $w \neq 0$,

$$
w \frac{d^{2} w}{d v^{2}}+\left(\frac{d w}{d v}\right)^{2}+\frac{1}{2} v \frac{d w}{d v}-4 \varepsilon \frac{d w}{d v}=0
$$


In terms of the new variables, the first order prolonged operator $\widetilde{\Xi}_{2}$ assumes the form

$$
\widetilde{\Xi}_{2}=(v-8 \varepsilon) \frac{\partial}{\partial v}+2 w \frac{\partial}{\partial w}
$$

by computing the new independent and dependent variables $r$ and s, respectively, such that

$$
\widetilde{\Xi}_{2}(r)=1, \quad \widetilde{\Xi}_{2}(s)=0,
$$

we have

$$
r=\log v_{0}+\varepsilon\left(\frac{v_{1}}{v_{0}}-\frac{8}{v_{0}}\right), \quad s=\frac{w_{0}}{v^{2}}+\varepsilon\left(16 \frac{w_{0}}{v^{3}}+\frac{w_{1}}{v^{2}}\right) .
$$

By inserting (100) in (97), we obtain

$$
s \frac{d^{2} s}{d r^{2}}+7 s \frac{d s}{d r}+\left(\frac{d s}{d r}\right)^{2}+\frac{1}{2} \frac{d s}{d r}+6 s^{2}+s=0 .
$$

Finally, by setting $\frac{d s}{d r}=p(s)$, the first order ordinary differential equation

$$
p s \frac{d p}{d s}+p^{2}+7 p s+\frac{1}{2} p+6 s^{2}+s=0
$$

is recovered. We notice that the small parameter $\varepsilon$ does not appear explicitly, but it is somehow hidden inside the involved variables.

Remark 3. The reduction of the perturbed Blasius equation to a first order ordinary differential equation where $\varepsilon$ does not appear explicitly, as well as the form of the admitted approximate Lie symmetries, suggests us the possibility of transforming equation (91) to the standard Blasius equation. Let us introduce the new independent and dependent variables $t$ and $v$, respectively, such that

$$
\Xi_{4}(t) \approx t, \quad \Xi_{4}(v) \approx-v
$$

whence

$$
v=u+\varepsilon\left(\frac{2}{3} x u-8\right), \quad t=x-\varepsilon \frac{x^{2}}{3},
$$

and equation (91) writes as

$$
\frac{d^{3} v}{d t^{3}}+\frac{1}{2} v \frac{d^{2} v}{d t^{2}}=0
$$

i.e., the classical Blasius equation. 


\subsection{Approximately invariant solutions of partial differential equa- tions}

In this Subsection we consider some examples of partial differential equations and compute some approximately invariant solutions.

Example 11. Consider the nonlinear wave equation

$$
\frac{\partial^{2} u}{\partial t^{2}}-\frac{\partial}{\partial x}\left(u^{2} \frac{\partial u}{\partial x}\right)+\varepsilon \frac{\partial u}{\partial t}=0
$$

The first order approximate symmetries are generated by the following vector fields:

$$
\begin{array}{ll}
\Xi_{1}=\frac{\partial}{\partial t}, & \Xi_{2}=\frac{\partial}{\partial x}, \\
\Xi_{3}=\left(t+\varepsilon \frac{t^{2}}{6}\right) \frac{\partial}{\partial t}-\left(u_{0}+\varepsilon\left(u_{1}+\frac{t u_{0}}{3}\right)\right) \frac{\partial}{\partial u}, & \Xi_{4}=x \frac{\partial}{\partial x}+\left(u_{0}+\varepsilon u_{1}\right) \frac{\partial}{\partial u}, \\
\Xi_{5}=\varepsilon \frac{\partial}{\partial t}, & \Xi_{6}=\varepsilon \frac{\partial}{\partial x}, \\
\Xi_{7}=\varepsilon\left(t \frac{\partial}{\partial t}-u_{0} \frac{\partial}{\partial u}\right), & \Xi_{8}=\varepsilon\left(x \frac{\partial}{\partial x}+u_{0} \frac{\partial}{\partial u}\right) .
\end{array}
$$

The solutions that result approximately invariant with respect to the Lie generator $\Xi_{3}$ are such that

$$
\left(t+\varepsilon \frac{t^{2}}{6}\right) \frac{\partial u}{\partial t}=-u_{0}-\varepsilon\left(u_{1}+\frac{t u_{0}}{3}\right)
$$

whereupon, insertion of $u=u_{0}+\varepsilon u_{1}+O\left(\varepsilon^{2}\right)$, and separation of the coefficients of different powers of $\varepsilon$, provide the system

$$
\left\{\begin{array}{l}
t \frac{\partial u_{0}}{\partial t}=-u_{0} \\
t \frac{\partial u_{1}}{\partial t}+\frac{t^{2}}{6} \frac{\partial u_{0}}{\partial t}=-u_{1}-\frac{t u_{0}}{3}
\end{array}\right.
$$

whose solution is

$$
u_{0}(t, x)=\frac{U_{0}(x)}{t}, \quad u_{1}(t, x)=\frac{U_{1}(x)}{t}-\frac{U_{0}(x)}{6} .
$$


Substitution of (109) into equation (106) provides the following reduced system of ordinary differential equations:

$$
\begin{aligned}
& \left(U_{0}^{2} U_{0}^{\prime}\right)^{\prime}-2 U_{0}=0 \\
& \left(U_{0}^{2} U_{1}\right)^{\prime \prime}-2 U_{1}=0
\end{aligned}
$$

where $U_{0}(x)$ and $U_{1}(x)$ are functions to be determined, and the prime' denotes differentiation with respect to $x$. A solution to the system (110) is

$$
U_{0}(x)= \pm x, \quad U_{1}(x)=\frac{k_{1}}{x^{3}}+k_{2}
$$

providing the following approximately invariant solution of (106):

$$
u(t, x)= \pm \frac{x}{t}+\varepsilon\left(\frac{k_{1}}{t x^{3}}+\frac{k_{2}}{t} \mp \frac{x}{6}\right) .
$$

Remark 4. In [28], equation (106) has been analyzed by means of the BaikovGazizov-Ibragimov approach for approximate symmetries. The solution there obtained that is approximately invariant with respect to the first order approximate symmetry generated by

$$
X=\left(t+\varepsilon \frac{t^{2}}{6}\right) \frac{\partial}{\partial t}-\left(u+\varepsilon \frac{t u}{3}\right) \frac{\partial}{\partial u}
$$

was

$$
u(t, x)= \pm\left(\frac{x}{t}-\varepsilon \frac{x}{6}\right),
$$

that is less general than (112). The approximate Lie generator (113) has the invariants

$$
J_{1}=x, \quad J_{2}=t u+\varepsilon \frac{t^{2} u}{6},
$$

and the approximately invariant solutions are sought by setting $J_{2}=\varphi\left(J_{1}\right)$, where the function $\varphi$ is determined by satisfying equation (106). The solution (112) could be recovered by setting $J_{2}=\varphi\left(J_{1}, \varepsilon\right)$ and, then, taking into account the expansion of $u$.

Example 12. Consider the Korteweg-deVries-Burgers equation

$$
\frac{\partial u}{\partial t}+u \frac{\partial u}{\partial x}-\frac{\partial^{2} u}{\partial x^{2}}+\varepsilon \frac{\partial^{3} u}{\partial x^{3}}=0
$$


and compute the solutions that are approximately invariant with respect to the Lie generator $\Xi=\Xi_{1}+c \Xi_{2}+\Xi_{7}$ (see (90)), where $c$ is a constant, i.e.,

$$
(1+2 \varepsilon t) \frac{\partial u}{\partial t}+(c+\varepsilon x) \frac{\partial u}{\partial x}=-\varepsilon u_{0}
$$

Therefore, inserting $u=u_{0}+\varepsilon u_{1}+O\left(\varepsilon^{2}\right)$, we get the system

$$
\left\{\begin{array}{l}
\frac{\partial u_{0}}{\partial t}+c \frac{\partial u_{0}}{\partial x}=0 \\
\frac{\partial u_{1}}{\partial t}+c \frac{\partial u_{1}}{\partial x}+2 t \frac{\partial u_{0}}{\partial t}+x \frac{\partial u_{0}}{\partial x}=-u_{0}
\end{array}\right.
$$

whose integration provides

$$
\left\{\begin{array}{l}
u_{0}(t, x)=U_{0}(\omega) \\
u_{1}(t, x)=\left(\frac{3 c t^{2}}{2}-t x\right) U_{0}^{\prime}(\omega)-t U_{0}(\omega)+U_{1}(\omega)
\end{array}\right.
$$

where $\omega=x-c t$, and $U_{0}(\omega), U_{1}(\omega)$ satisfy the following reduced system of ordinary differential equations:

$$
\begin{aligned}
& U_{0}^{\prime \prime}+\left(U_{0}-c\right) U_{0}^{\prime}=0, \\
& \left(U_{1}^{\prime}+\left(c-U_{0}\right)\left(U_{1}+U_{0}^{\prime}\right)+\omega U_{0}\right)^{\prime}=0
\end{aligned}
$$

the prime' denoting the differentiation with respect to $\omega$; by solving system (114), we find the following first order approximately invariant solution

$$
\begin{aligned}
& u(t, x)=c-2 k_{1} \tanh \left(k_{1} \omega+k_{2}\right) \\
& \quad+\varepsilon\left(k_{4} \operatorname{sech}^{2}\left(k_{1} \omega+k_{2}\right)+\left(( c + 4 k _ { 1 } ^ { 2 } \omega ) \operatorname { c o s h } \left(\left(2\left(k_{1} \omega+k_{2}\right)\right)\right.\right.\right. \\
& \quad-\left(4\left(k_{2} c-k_{1} k_{3}\right)\left(k_{1} \omega+k_{2}\right)+2 k_{1}^{2} c \omega^{2}-32 k_{1}^{4} \log \left(\cosh \left(k_{1} \omega+k_{2}\right)\right)\right. \\
& \left.\quad+2\left(k_{1}\left(1-k_{3}\right)+c\left(k_{1} \omega+k_{2}\right)\right) \sinh \left(2\left(k_{1} \omega+k_{2}\right)\right)\right) \frac{\operatorname{sech}^{2}\left(k_{1} \omega+k_{2}\right)}{8 k_{1}^{2}}
\end{aligned}
$$

where $k_{1}, k_{2}, k_{3}$ and $k_{4}$ are constants. 


\section{Conclusions}

In this paper, we proposed a new approach to approximate Lie symmetries of differential equations. Such a method combines the elegance of the approach by Baikov, Gazizov and Ibragimov [27] with the requirements of perturbative analysis of differential equations. Remarkably, it allows to use all the techniques of classical Lie group analysis in an approximate context.

The computational cost in order to determine the approximate symmetries of a differential equation using the method proposed here is higher than that of the classical Lie group analysis; nevertheless, a working Reduce [56] package doing automatically all the needed work is available [57].

These approximate symmetries can be used to lower the order of ordinary differential equations as well as to compute approximately invariant solutions of partial differential equations. Further applications for deriving approximate conservation laws, or local transformations (suggested by the approximate symmetries) mapping differential equations to approximately equivalent ones are possible. Moreover, either approximate equivalence transformations [1, 58, 59, 60] for classes of differential equations involving small terms or approximate conditional symmetries can be defined [61, 62, 63]. Some of these extensions are currently under investigation. As a final comment, we observe that this approach can be generalized to include multiple scales [64, 65] in the independent variables in order to avoid the occurrence of secular-like terms in the solutions. These extensions and generalizations will be the object of forthcoming papers.

\section{Acknowledgments}

Work supported by G.N.F.M. of I.N.d.A.M. and by local grants of the University of Messina. The authors thank the referees for their useful comments.

\section{References}

[1] Ovsiannikov, L. V.: Group analysis of differential equations. Academic Press, New York (1982).

[2] Ibragimov, N. H.: Transformation groups applied to mathematical physics. D. Reidel Publishing Company, Dordrecht (1985). 
[3] Olver, P. J.: Applications of Lie groups to differential equations. Springer, New York (1986).

[4] Ibragimov, N. H., editor, CRC Handbook of Lie group analysis of differential equations (three volumes). CRC Press, Boca Raton (1994, 1995, 1996).

[5] Olver, P. J.: Equivalence, invariants, and symmetry. Cambridge University Press, New York (1995).

[6] Baumann, G.: Symmetry analysis of differential equations with Mathematica. Springer, New York (2000).

[7] Bluman, G. W., Anco, S. C.: Symmetry and integration methods for differential equations. Springer, New York (2002).

[8] Meleshko, S. V.: Methods for constructing exact solutions of partial differential equations. Springer, New York (2005).

[9] Bluman, G. W., Cheviakov, A. F., Anco, S. C.: Applications of symmetry methods to partial differential equations. Springer, New York (2009).

[10] Bordag, L. A.: Geometrical properties of differential equations. Applications of the Lie group analysis in Financial Mathematics. World Scientific, Singapore (2015).

[11] Oliveri, F., Speciale, M. P: Exact solutions to the equations of ideal gasdynamics by means of the substitution principle, Int. J. Non-linear Mech., 33, 585-592 (1998).

[12] Oliveri, F., Speciale, M. P: Exact solutions to the equations of perfect gases through Lie group analysis and substitution principles, Int. J. Non-linear Mech., 34, 1077-1087 (1999).

[13] Oliveri, F., Speciale, M. P: Exact solutions to the unsteady equations of perfect gases through Lie group analysis and substitution principles, Int. J. Nonlinear Mech., 37, 257-274 (2002).

[14] Oliveri, F.: On substitution principles in ideal magneto-gasdynamics by means of Lie group analysis, Nonlinear Dyn., 42, 217-231 (2005). 
[15] Oliveri, F., Speciale, M. P: Exact solutions to the ideal magneto-gasdynamics equations through Lie group analysis and substitution principles, J. Phys. A: Math. Gen., 38, 8803-8820 (2005).

[16] Bluman, G. W., Kumei, S.: Symmetries and differential equations. Springer, New York (1989).

[17] Kumei, S., Bluman, G. W.: When nonlinear differential equations are equivalent to linear differential equations, SIAM J. Appl. Math., 42, 1157-1173 (1982).

[18] Donato, A., Oliveri, F.: Reduction to autonomous form by group analysis and exact solutions of axi-symmetric MHD equations, Math. Comput. Modelling, 18, 83-90 (1993).

[19] Donato, A., Oliveri, F.: Linearization procedure of nonlinear first order systems of PDE's by means of canonical variables related to Lie groups of point transformations, J. Math. Anal. Appl., 188, 552-568 (1994).

[20] Donato, A., Oliveri, F.: When nonautonomous equations are equivalent to autonomous ones, Applicable Analysis, 58, 313-323 (1995).

[21] Donato, A., Oliveri, F.: How to build up variable transformations allowing one to map nonlinear hyperbolic equations into autonomous or linear ones, Transp. Th. Stat. Phys., 25, 303-322 (1996).

[22] Currò, C., Oliveri, F.: Reduction of nonhomogeneous quasilinear $2 \times 2$ systems to homogeneous and autonomous form, J. Math. Phys., 49, 103504-1103504-11 (2008).

[23] Oliveri, F.: Lie symmetries of differential equations: classical results and recent contributions, Symmetry, 2, 658-706 (2010).

[24] Oliveri, F.: General dynamical systems described by first order quasilinear PDEs reducible to homogeneous and autonomous form, Int. J. Non-linear Mech., 47, 53-60 (2012).

[25] Gorgone, M., Oliveri, F.: Nonlinear first order partial differential equations reducible to first order homogeneous and autonomous quasilinear ones, Ricerche Mat., 66, 51-63 (2017). 
[26] Gorgone, M., Oliveri, F.: Nonlinear first order PDEs reducible to autonomous form polynomially homogeneous in the derivatives, J. Geom. Phys., 113, 53-64 (2017).

[27] Baikov, V. A., Gazizov, R. I., Ibragimov, N. Kh.: Approximate symmetries, Mat. Sb., 136, 435-450 (1988); English Transl. in Math. USSR Sb., 64, 427441 (1989).

[28] Ibragimov, N. H., Kovalev, V. K.: Approximate and renormgroup symmetries. Higher Education Press, Beijing and Springer-Verlag GmbH, BerlinHeidelberg (2009).

[29] Wiltshire, R. J.: Perturbed Lie symmetry and systems of non-linear diffusion equations, Nonlinear Math. Phys., 3, 130-138 (1996).

[30] Kovalev, V. F.: Approximate transformation groups and renormgroup symmetries, Nonlinear Dyn., 22, 73-83 (2000).

[31] Baikov, V. A., Kordyukova, S. A.: Approximate symmetries of the Boussinesq equation, Quaestiones Mathematicae, 26, 1-14 (2003).

[32] Dolapçi, I. T., Pakdemirli, M.: Approximate symmetries of creeping flow equations of a second grade fluid, Int. J. Non-linear Mech., 39, 1603-1618 (2004).

[33] Pakdemirli, M., Yürüsoy, M., Dolapçi, I. T.: Comparison of approximate symmetry methods for differential equations, Acta Appl. Math., 80, 243271 (2004).

[34] Ibragimov, N. H., Ünal, G., Jogréus, C.: Approximate symmetries and conservation laws for Itô and Stratonovich dynamical systems, J. Math. Anal. Appl., 297, 152-168 (2004).

[35] Wiltshire, R.: Two approaches to the calculation of approximate symmetry exemplified using a system of advection-diffusion equations, J. Comp. Appl. Math., 197, 287-301 (2006).

[36] Kara, A.H., Mahomed, F.M., Qadir, A.: Approximate symmetries and conservation laws of the geodesic equations for the Schwarzschild metric, Nonlinear Dyn., 51, 183-188 (2008). 
[37] Gazizov, R. K., Ibragimov, N. H., Lukashchuk, V. O.: Integration of ordinary differential equation with a small parameter via approximate symmetries: reduction of approximate symmetry algebra to a canonical form, Lobachevskii J. Math., 31, 141-151 (2010).

[38] Gan, Y., Qu, C.: Approximate conservation laws of perturbed partial differential equations, Nonlinear Dyn., 61, 217-228 (2010).

[39] Gazizov, R. K., Ibragimov, N. H.: Approximate symmetries and solutions of the Kompaneets equation, J. Appl. Mech. Techn. Phys., 55, 220-224 (2014).

[40] Nayfeh, A. H.: Introduction to Perturbation Techniques. Wiley, New York (1981).

[41] Donato, A., Palumbo, A.: Approximate invariant solutions to dissipative systems. In: Nonlinear waves and dissipative effects. Research Notes, PitmanLongman, 66-75 (1991).

[42] Donato, A., Palumbo, A.: Approximate asymptotic symmetries, Comput. Appl. Math., II, W. F. Ames and P. J. van der Houwen eds., Elsevier Science Publishers B. V., North-Holland, 141-151 (1992).

[43] Fushchich, W. I., Shtelen, W. H.: On approximate symmetry and approximate solutions of the non-linear wave equation with a small parameter, J. Phys. A: Math. Gen., 22, 887-890 (1989).

[44] Euler, N., Shulga, M. W., Steeb, W. H.: Approximate symmetries and approximate solutions for a multi-dimensional Landau-Ginzburg equation, J. Phys. A: Math. Gen., 25, 1095-1103 (1992).

[45] Euler, M., Euler, N., Köhler, A.: On the construction of approximate solutions for a multidimensional nonlinear heat equation, J. Phys. A: Math. Gen., 27, 2083-2092 (1994).

[46] Euler, N., Euler, M.: Symmetry properties of the approximations of multidimensional generalized Van der Pol equations, J. Nonlinear Math. Phys., 1, 41-59 (1994).

[47] Diatta, B. Wafo Soh, C., Khalique, C. M.: Approximate symmetries and solutions of the hyperbolic heat equation, Appl. Math. Comp., 205, 263-272 (2008). 
[48] Hereman, W.: Review of symbolic software for the computation of Lie symmetries of differential equations, Mathl. Comput. Modelling, 25, 115-132 (1997).

[49] Butcher, J., Carminati, J., Vu, K.T.: A comparative study of some computer algebra packages which determine the Lie point symmetries of differential equations, Comput. Phys. Comm., 155, 92-114 (2003).

[50] Cheviakov. A. F.: Symbolic computation of local symmetries of nonlinear and linear partial and ordinary differential equations, Math. Comput. Sci., 4, 203-222 (2010).

[51] Jefferson, G. F., Carminati, J.: ASP: Automated symbolic computation of approximate symmetries of differential equations, Comput. Phys. Comm., 184, 1045-1063 (2013).

[52] Vu, K.T., Jefferson, G. F., Carminati, J.: Finding higher symmetries of differential equations using the MAPLE package DESOLVII, Comput. Phys. Comm., 183, 1044-1054 (2012).

[53] Valenti, A.: Approximate symmetries for a model describing dissipative media, Proceedings of 10th International Conference in Modern Group Analysis (Larnaca, Cyprus), 236-243 (2005).

[54] Ruggieri, M., Speciale, M. P.: Approximate symmetries in viscoelasticity, Theor. Math. Phys., 189, 1500-1508 (2016).

[55] Ruggieri, M., Speciale, M. P.: Lie group analysis of a wave equation with a small nonlinear dissipation, Ricerche Mat., 66, 27-34 (2017).

[56] Hearn, A. C.: Reduce Users' Manual Version 3.8. Santa Monica, CA, USA (2004).

[57] Oliveri, F.: ReLie: a Reduce package for Lie group analysis of differential equations. Submitted, 2017.

[58] Lisle, I. G.: Equivalence transformations for classes of differential equations. $\mathrm{PhD}$ dissertation, University of British Columbia, Vancouver, BC, Canada (1992). Available at the URL https://open.library.ubc.ca/cIRcle/collections/ubctheses/831/items/1.0079820 
[59] Meleshko, S. V.: Generalization of the equivalence transformations, J. Nonlinear Math. Phys., 3, 170-174 (1996).

[60] Gorgone, M., Oliveri, F., Speciale, M. P.: Reduction of balance Laws in (3+ 1)-dimensions to autonomous conservation laws by means of equivalence transformations, Acta Appl. Math., 132, 333-345 (2014).

[61] Bluman, G. W., Cole, J. D.: The general similarity solution of the heat equation, J. Math. Mech., 18, 1025-1042 (1969).

[62] Arrigo, D. J., Broadbridge, P., Hill, J. M.: Nonclassical symmetry solutions and the methods of Bluman-Cole and Clarkson-Kruskal, J. Math. Phys., 34, 4692-4703 (1993).

[63] Cerniha, R.: New Q-conditional symmetries and exact solutions of some reaction-diffusion- convection equations arising in mathematical biology, $\mathrm{J}$. Math. Anal. Appl., 326, 783-799 (2007).

[64] Baikov, V. A., Ibragimov, N.H.: Continuation of approximate transformation groups via multiple time scales method, Nonlinear Dyn., 22, 3-13 (2000).

[65] Kordyukova, S. A.: Approximate group analysis and multiple time scales method for the approximate Boussinesq equation, Nonlinear Dyn., 46, 7385 (2006). 